\title{
Lattice study of finite volume effect in HVP for muon g-2
}

\author{
Taku Izubuchi ${ }^{1,2}$, Yoshinobu Kuramashi ${ }^{3,4}$, Christoph Lehner ${ }^{2}$, and Eigo Shintani ${ }^{4, \star}$ \\ ${ }^{1}$ RIKEN-BNL Research Center, Brookhaven National Laboratory, Upton, NY 11973, USA. \\ ${ }^{2}$ High Energy Theory Group, Brookhaven National Laboratory, Upton, NY 11973, USA. \\ ${ }^{3}$ Center for Computational Sciences, University of Tsukuba, Tsukuba, Ibaraki 305-8577, Japan. \\ ${ }^{4}$ RIKEN Advanced Institute for Computational Science, Kobe, Hyogo 650-0047, Japan.
}

\begin{abstract}
We study the finite volume effect of the hadronic vacuum polarization contribution to muon $\mathrm{g}-2, a_{\mu}^{\text {hvp }}$,in lattice QCD by comparison with two different volumes, $L^{4}=(5.4)^{4}$ and $(8.1)^{4} \mathrm{fm}^{4}$, at physical pion. We perform the lattice computation of highly precise vector-vector current correlator with optimized AMA technique on $N_{f}=2+1$ PACS gauge configurations in Wilson-clover fermion and stout smeared gluon action at one lattice cut-off, $a^{-1}=2.33 \mathrm{GeV}$. We compare two integrals of $a_{\mu}^{\text {hvp }}$, momentum integral and time-slice summation, on the lattice and numerically show that the different size of finite volume effect appears between two methods. We also discuss the effect of backward-state propagation into the result of $a_{\mu}^{\text {hvp }}$ with the different boundary condition. Our model-independent study suggest that the lattice computation at physical pion is important for correct estimate of finite volume and other lattice systematics in $a_{\mu}^{\text {hvp }}$.
\end{abstract}

\section{Introduction}

The muon anomalous magnetic moment (g-2) is an essential observable for a rigorous test of the standard model (SM). The experimental value of muon $\mathrm{g}-2$ has been measured a couple of decades ago in BNL E821 experiment, and the value $a_{\mu}^{\mathrm{E} 821}=11659209.1(5.4)(3.3) \times 10^{-10}$ has been the highest precision of muon g-2 measurement [1,2]. On the other hand, by the recent development of QED loop computation at 5-loop order [3], its accuracy is achieved to $O\left(10^{2}\right)$ magnitude higher than $a_{\mu}^{\mathrm{E} 821}$. While the electroweak interaction contribution involving $W^{ \pm}, Z$ and Higgs is sub-leading order and its precision is an $O(10)$ magnitude higher than $a_{\mu}^{\mathrm{E} 821}$, the leading order of QCD contribution, computed from phenomenological description of hadronic vacuum polarization (HVP) with $e^{+} e^{-} \rightarrow$ hadron cross section or $\tau \rightarrow$ hadron decay, has a large uncertainty similar to error of $a_{\mu}^{\mathrm{E} 821}$. Moreover the next-to-leading order of QCD contribution, mainly light-by-light contribution, is still under discussion about a possible uncertainty due to model dependence [4]. To resolve the issue of so-called muon g-2 anomaly, which is a discrepancy between experiment and the SM calculation currently being 2.4-3.6 $\sigma$, we need to reduce uncertainties of both QCD and experiment as possible. The upcoming experiment in FermiLab [5] and J-PARC [6] is aiming for a factor 4 improvement from $a_{\mu}^{\mathrm{E} 821}$ up to the next decade, and so that the same order of precision should be achieved even from the theoretical side.

\footnotetext{
${ }^{\star}$ Speaker, e-mail: shintani@ riken.jp
} 
In this proceedings we focus on the lattice QCD computation of $a_{\mu}^{\text {hvp }}$ using connected HVP diagram on two volumes, $L=5.4$ and $8.1 \mathrm{fm}$, at physical pion $\left(m_{\pi} \simeq 0.14 \mathrm{GeV}\right)$, namely there are two variations of $m_{\pi} L=3.8$ and 5.8, in order for a solid study of finite volume effect in lattice QCD without effective models. Our lattice setup is not necessary to take the chiral extrapolation, and thus it is not only a removal of uncertainty of chiral extrapolation but also possible to straightforwardly compare finite volume effect in infrared region on two different lattice sizes. Although this enables us to perform a rigorous test of lattice study of finite volume effect, the statistical noise apparently matters, especially in infrared region [7]. To overcome such an issue, we utilize highly optimized AMA techniques reported in [8] to significantly reduce the computational cost rather than original proposals [9-11]. It is successful to be achieved into sufficiently high precision to realize the discrepancy between two volumes in infrared region. Furthermore reducing the computational cost, we can also precisely investigate the boundary effect, which is regarded as one of the finite volume effects, into integrand of $a_{\mu}^{\text {hvp }}$. Our study provides an essential instruction for the future lattice QCD computation to reach sub-percent level of $a_{\mu}^{\text {hvp }}$.

\section{Lattice computation of $a_{\mu}^{\text {hvp }}$}

Since the lattice QCD calculation is defined on Euclidean space-time, conventional representation of $a_{\mu}^{\text {hvp }}$ as the integral of vacuum polarization function (VPF) $\Pi(Q)$ with respect to Euclidean momentum squared $Q^{2}$ from zero to infinity,

$$
\begin{aligned}
& a_{\mu}^{\mathrm{hvp}}=\left(\frac{\alpha_{e}}{\pi}\right)^{2} \int_{0}^{\infty} d Q^{2} K_{E}\left(Q^{2}\right) \hat{\Pi}\left(Q^{2}\right), \quad \hat{\Pi}\left(Q^{2}\right) \equiv \Pi\left(Q^{2}\right)-\Pi(0), \\
& K_{E}(s)=\frac{1}{m_{\mu}^{2}} \hat{s} Z^{3}(\hat{s}) \frac{1-\hat{s} Z(\hat{s})}{1+\hat{s} Z^{2}(\hat{s})}, \quad Z(\hat{s})=-\frac{\hat{s}-\sqrt{\hat{s}^{2}+4 \hat{s}}}{2 \hat{s}}, \quad \hat{s}=\frac{s}{m_{\mu}^{2}},
\end{aligned}
$$

which is derived as a consequence of the analytic continuation from original representation in timelike momentum $q^{2}\left(=-Q^{2}\right)[12,13]$, is useful. $K_{E}$ has been known as the QED kernel given from oneloop computation, and $\alpha_{e}$ is a fine structure constant, $\alpha_{e}=1 / 137.03599914$. In this representation, $\hat{\Pi}$ denotes the renormalized VPF at $Q^{2}=0$, and so that this integral is converged into finite number. On the lattice computation of $a_{\mu}^{\text {hvp }}$, since the lattice data is discretized due to a finite lattice spacing on a finite box, basically we convert its integral to the definite summation up to a edge of lattice. In this paper we use two different ways to evaluate Eq. (1) on the lattice; one is the direct $Q^{2}$ integral of Eq. 1 using the continuous function of VPF obtained by fitting lattice data, and another one is an time-slice summation of vector-vector current correlator on the lattice using the conversion of Eq.(1) into Euclidean coordinate space-time, so called "time-momentum representation" (TMR) [14]. We will define each representation below.

\subsection{Momentum integral}

First, we briefly introduce the traditional method to evaluate Eq. 1 from VPF. The VPF is evaluated from the Fourier transformation of vector-vector current correlator,

$$
\sum_{x}\left\langle V_{\mu}^{\mathrm{EM}}(x) V_{v}^{\mathrm{EM}}(0)\right\rangle e^{i Q x}=\left(Q^{2} \delta_{\mu v}-Q_{\mu} Q_{v}\right) \Pi(Q), \quad V_{\mu}^{\mathrm{EM}}(x)=\sum_{q} e_{q} V_{\mu}^{q}(x),
$$

with quark electric charge, $e_{q}=(u, d, s, \cdots)$. In this paper, we use three quark flavors, $q=(u, d, s)$. Lattice momentum is discretized as $Q_{\mu}=2 \pi n_{\mu} / L_{\mu}, n_{\mu} \in\left(-L_{\mu} / 2, L_{\mu} / 2\right]$. Since the local vector current, 
$V_{\mu}^{\text {loc }}=Z_{V} \bar{q}(x) \gamma_{\mu} q(x)$, on the lattice does not satisfy the Ward-Takahashi identity, the point-splitting current form,

$$
V_{\mu}^{\mathrm{cv}}(x)=\frac{1}{2}\left[\bar{q}(x+a \hat{\mu})\left(1+\gamma_{\mu}\right) U_{\mu}^{\dagger}(x) q(x)-\bar{q}(x)\left(1-\gamma_{\mu}\right) U_{\mu}(x) q(x+a \hat{\mu})\right],
$$

can be defined as the conserved current. As used in [7, 15, 16], we employ the vector-vector current correlator composed of the combination of conserved and local current. It is convenient to avoid contact term [15], while its Ward-Takahashi identity is partially conserved. In order to correct such a non-conservation, the $\mathrm{Z}$ factor, $Z_{V}=0.95153(76)(1487)$ evaluated from Schrödinger functional method [17] is used. In this paper we only compute the connected diagram.

For the evaluation of $a_{\mu}$ from the integral of Eq. (1), the fit function with Padé approximation [18],

$$
\Pi^{\mathrm{fit}}(Q)=C+P_{[n, m]}\left(Q^{2}\right), \quad P_{[n, m]}=Q^{2}\left(A_{0} \delta_{n, m+1}+\sum_{k=1}^{m} \frac{A_{k}}{Q^{2}+B_{k}}\right),
$$

with the same convention as [7] is commonly used as model independent way. The parameters $A_{k}$, $B_{k}$ govern the dependence of $Q^{2}$ and $C$ is extrapolated value of renormalization constant $\Pi(0)$. This ansatz is used for a convergence test as increase of the order of $[n, m]$, which corresponds to the number of pole contributing to low- $Q^{2}$ region.

\subsection{Time-slice summation}

Using the vector-vector current correlator at zero momentum in spatial direction $i$,

$$
C(t)=\int d^{3} \vec{x}\left\langle V_{i}^{\mathrm{cv}}(\vec{x}, t) V_{i}^{\mathrm{loc}}(0)\right\rangle,
$$

$a_{\mu}^{\text {hvp }}$ in Eq. (1) can be also represented as

$$
a_{\mu}^{\mathrm{hvp}}=4 \alpha_{e}^{2} m_{\mu} \int_{0}^{\infty} d t t^{3} C(t) \tilde{K}(t), \quad \tilde{K}(t)=\frac{2}{m_{\mu} t^{3}} \int_{0}^{\infty} \frac{d \omega}{\omega} K_{E}\left(\omega^{2}\right)\left[\omega^{2} t^{2}-4 \sin ^{2}(\omega t / 2)\right],
$$

as shown in [14]. On the lattice, however, $C(t)$ is obtained at a discretized time-slice in the lattice unit $a$ limited to finite temporal length $N_{t}$, it thus becomes summation of $C(t)$ multiplied with $\tilde{K}(t)$ up to a half length of lattice temporal extension ${ }^{1}$. Setting the cutting edge $t_{\text {cut }}<N_{t} a / 2$ in an integral of Eq. 7, the lattice representation is

$$
\left[a_{\mu}^{\mathrm{hvp}}\right]_{\mathrm{lat}}\left(t_{\mathrm{cut}}\right) \equiv \sum_{t / a=0}^{t_{\mathrm{cut}} / a} C(t) W_{t}(t), \quad W_{t}(t)=8 \alpha_{e}^{2} \int_{0}^{\infty} \frac{d \omega}{\omega} K_{E}\left(\omega^{2}\right)\left[\omega^{2} t^{2}-4 \sin ^{2}(\omega t / 2)\right],
$$

in which the expression of $\tilde{K}$ in Eq. (7) is substituted.

We also note that the representation of Eq. (8) is not unique on the lattice since the lattice momentum is not uniquely defined. If we use sin functional form of lattice momentum, $\tilde{Q}=2 a^{-1} \sin \left(Q_{\mu} / 2 a\right)$, such a representation is changed to

$$
\left[\tilde{a}_{\mu}^{\mathrm{hvp}}\right]_{\mathrm{lat}}\left(t_{\mathrm{cut}}\right)=8 \alpha_{e}^{2} \sum_{t / a=0}^{t_{\mathrm{cut}} / a} C(t) \int_{0}^{\infty} \frac{\omega d \omega}{\tilde{\omega}^{2}} K_{E}\left(\omega^{2}\right)\left[\tilde{\omega}^{2} t^{2}-4 \sin ^{2}(\omega t / 2)\right],
$$

where we use $\tilde{\omega}=2 a^{-1} \sin (a Q / 2)$. The trivial difference between Eq. (8) and Eq. (9) is at $t=a$, in which the integrand of $\left[\tilde{a}_{\mu}^{\text {hvp }}\right]_{\text {lat }}$ is zero, besides that of $\left[a_{\mu}^{\text {hvp }}\right]_{\text {lat }}$ is non-zero. This discrepancy is regarded as the lattice artifact due to violating Lorentz symmetry on the lattice.

\footnotetext{
${ }^{1}$ In periodic or anti-periodic boundary condition, backward-state propagation significantly affects as finite volume correction. We numerically study in section 4 .
} 
Table 1. Table of parameters of PACS gauge ensembles. $L$ and $T$ denote spatial and temporal length. In Wilson-clover fermion, $K_{l}$ and $K_{s}$ denotes kappa values for light and strange quark flavors respectively.

\begin{tabular}{cccccccc}
\hline \hline$L / a$ & $T / a$ & $K_{l}$ & $K_{s}$ & $m_{\pi}(\mathrm{GeV})$ & $m_{K}(\mathrm{GeV})$ & $m_{\pi} L$ & configs. \\
\hline $96[8.1 \mathrm{fm}]$ & $96[8.1 \mathrm{fm}]$ & 0.126117 & 0.124790 & $0.1461(4)$ & $0.5242(3)$ & 6.0 & 50 \\
\hline $64[5.4 \mathrm{fm}]$ & $64[5.4 \mathrm{fm}]$ & 0.126117 & 0.124902 & $0.1385(9)$ & $0.5004(4)$ & 3.9 & 87 \\
\hline \hline
\end{tabular}

\section{Lattice set-up and its parameter}

In this proceedings, we use gauge configurations of non-perturbatively $O(a)$ improved Wilson fermion in $N_{f}=2+1$ on stout smeared gauge action with $\beta=1.82$ at physical point (see Table 1). PACS collaboration have generated it on two different volumes $L / a=64$ and 96 , corresponding to $5.4 \mathrm{fm}^{4}$ and $8.1 \mathrm{fm}^{3}$, at a cut-off scale $a^{-1}=2.332(18)[17,19]$.

In the measurement of vector-vector current correlator, we apply the AMA technique [9-11] to boost the statistical accuracy. AMA is defined with the master formula for the measurement of target observable $O$, which is vector-vector current correlator in this case,

$$
O^{\mathrm{AMA}}=\frac{1}{N_{\text {org }}} \sum_{f \in G}^{N_{\text {org }}}\left[O^{(\mathrm{org}) f}-O^{(\text {appx })}\right]+\frac{1}{N_{G}} \sum_{g \in G}^{N_{G}} O^{(\text {appx }) g},
$$

with covariant transformation $g \in G$ under subset of its symmetry $G$. Here $G$ corresponds to translational symmetry and its size is $N_{G}$ for approximation and $N_{\text {org }}$ for original. In [8], one of the authors have developed the highly optimized AMA using SAP deflation preconditioning [20, 21]. From the practical point of view, aiming for $N_{G} \sim O\left(10^{3}\right)$, we tune the parameter of approximation to be small $\Delta r$ [9-11], in which $O^{\text {(appx) }}$ and $O^{\text {(org) }}$ are strongly correlated, as $\Delta r \lesssim N_{G} / 2 \sim O\left(10^{-4}\right)$ for the scaling of statistical error close to $1 / \sqrt{N_{G}}$.

In the computation of $O^{\text {AMA }}$, we use a method with fixed number of iteration of General Conjugate Residual (GCR) solver with SAP deflation as used in [8]. In a generation of SAP deflation field, the domain block size, the number of SAP cycle $n_{\mathrm{cy}}$ and the number of deflation vector are tuned. SAP is used in not only preconditioning of GCR, but also generation of deflation field overlapping with low-mode dominance by smoothing technique (inexact deflation [21]).

\section{Numerical results}

\subsection{Analysis of vector-vector current correlator}

First, we show the time-slice dependence of vector-vector current correlator $C(t)$ from short to long distance in Figure 1. For the computation of $a_{\mu}^{\text {hvp }}$ using eq. (8), we need to know the precise behavior of $C(t)$ in the infrared region. Our high-statistics result boosted by AMA method show a clear signal beyond $t=2.5 \mathrm{fm}$, which is a temporal edge of $64^{4}$ lattice, and it thus enables us to compare between different volumes in the infrared region. On our gauge ensembles, the effective mass plot of $C(t)$ in right of Figure 1 shows the vector meson mass is smaller than rho meson mass at $t>1.2 \mathrm{fm}$. Since energy of two light pions $\left(m_{\pi}<0.145 \mathrm{GeV}\right)$ in large volume $(L=8.1 \mathrm{fm})$ is smaller than threshold of rho meson, such a state, which has the energy among $m_{\rho}>E_{\pi \pi}>2 \sqrt{m_{\pi}^{2}+(2 \pi / L)^{2}} \approx 0.421 \mathrm{GeV}$, may be a main contribution as the ground state in infrared region. It turns out that, on the gauge ensemble we used, $C(t)$ contains the contribution of not the asymptotic state of single particle but the multihadron state as a consequence of utilization of physical pion mass. We note that the effective mass at 

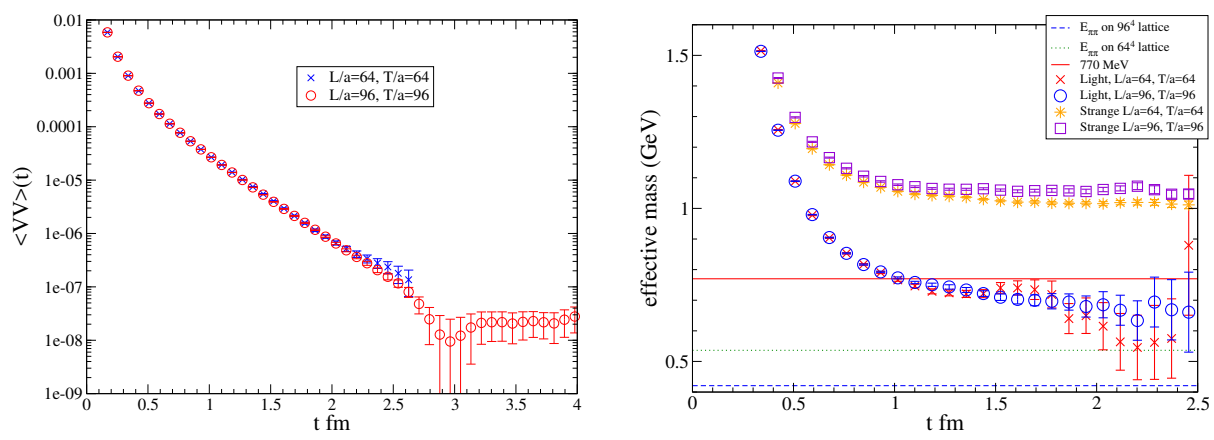

Figure 1. (Left) The vector-vector correlator as a function of time-slice in fm unit. (Right) Effective mass plot of vector-vector corrent correlator on each lattice box.

strange quark flavor on $L / a=96$ is slightly heavier than $L / a=64$, in which the kappa parameter for sea strange quark is set to being different (see Table 1).

\subsection{Computation of $a_{\mu}^{\text {hvp }}$ with time-slice summation}

In this section we investigate the volume dependence for the integrand of $\left[a_{\mu}^{\text {hvp }}\right]_{\text {lat }}$ (see Eq. (8)) and its time-slice summation. The integrand at time-slice $t$ and summation over its time-slice up to $t_{\text {cut }}$ of light quark contribution on each lattice volume is compared in Figure 2. Light quark contribution is dominated in $\left[a_{\mu}^{\text {hvp }}\right]_{\text {lat }}$ (strange contribution is a few percent magnitude for $\left[a_{\mu}^{\text {hvp }}\right]_{\text {lat }}$ as a consequence of its large mass and $1 / 5=\left(e_{s} / e_{l}\right)^{2}$ factor of electric charge ratio). Compared between $L / a=96$ and $L / a=64$ lattice, the integrand at light quark flavor has similar shape and its time-slice summation is not significantly different even at $t_{\text {cut }}=2.5 \mathrm{fm}$. We observe that FV correction on $L / a=64$ is similar order of magnitude as statistical fluctuation.

From Figure 3 we compare two sizes of temporal length to observe the effect of BSP on $L / a=64$ lattice. It significantly appears that from $t=2.4 \mathrm{fm}$ for integrand such a contribution to $\left[a_{\mu}^{\text {hvp }}\right]_{\text {lat }}$ is thus maximally $4 \%$ contribution at $t_{\text {cut }}=2.5 \mathrm{fm}$.

\subsection{Computation of $a_{\mu}^{\mathrm{hvp}}$ in $Q^{2}$ integral}

Figure 4 plots the lattice data of VPF and integrand as a function of $Q^{2}$ computed on $96^{4}$ and $64^{4}$ lattice. To obtain $\hat{\Pi}(Q)$, which is renormalized by subtraction to $\Pi(0)$, in Eq. (1), we perform the extrapolation for $\Pi(0)$ and interpolation between discretized $Q^{2}$ with a Padé ansatz. As used in Mainz group [7], we employ [2,1] degree of Padé ansatz to fit lattice VPF data. The chi-squared fitting with Padé ansatz is working well for both lattices. We observe that, in low- $Q^{2}$ below $Q^{2} \approx 0.3 \mathrm{GeV}^{2}$, the significant discrepancy of VPF at light quark flavor between $96^{4}$ and $64^{4}$ lattices appears, and its discrepancy then decreases as $Q^{2}$ increases. This may be due to FV effect in VPF since low- $Q^{2}$ region around hadronic scale $Q^{2} \sim \Lambda^{2}$ is suffered large FV correction.

\section{Discussion}

Figure 5 shows a summary plot of $a_{\mu}^{\text {hvp }}$ at light quark flavor as $m_{\pi} L$ dependence. FV effect will differently contribute between time-slice summation and $Q^{2}$ integral, where $Q^{2}$ integral may largely 

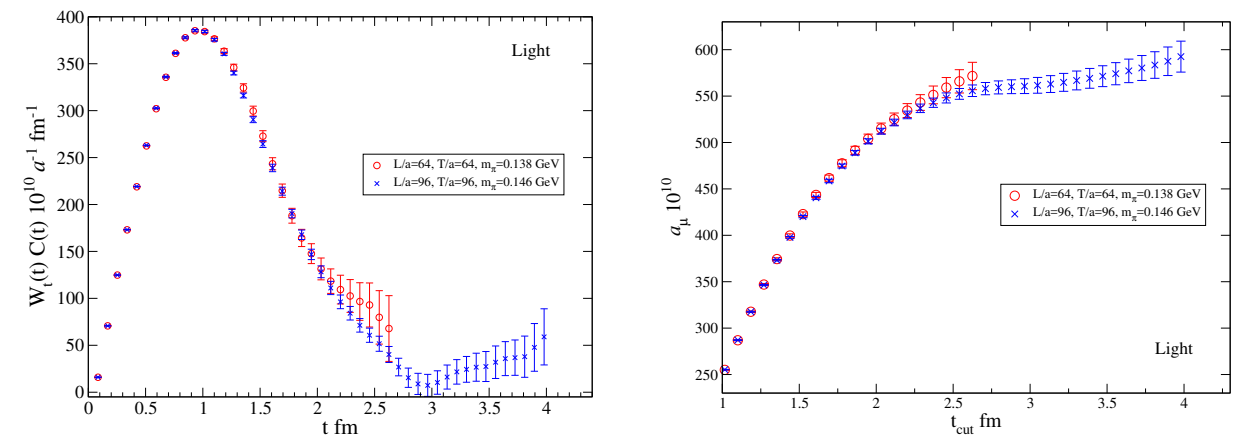

Figure 2. (Left) Integrand of $\left[a_{\mu}^{\text {hvp }}\right]_{\text {lat }}$ in Eq. (8) divided by lattice spacing as a function of time-slice in physical unit. Different symbols denote the results in each gauge ensemble at light flavor. (Right) Time-slice summation for $\left[a_{\mu}^{\text {hvp }}\right]_{\text {lat }}$ up to $t_{\text {cut }}$ at light quark flavor. Different symbols denote the data of each gauge ensemble.
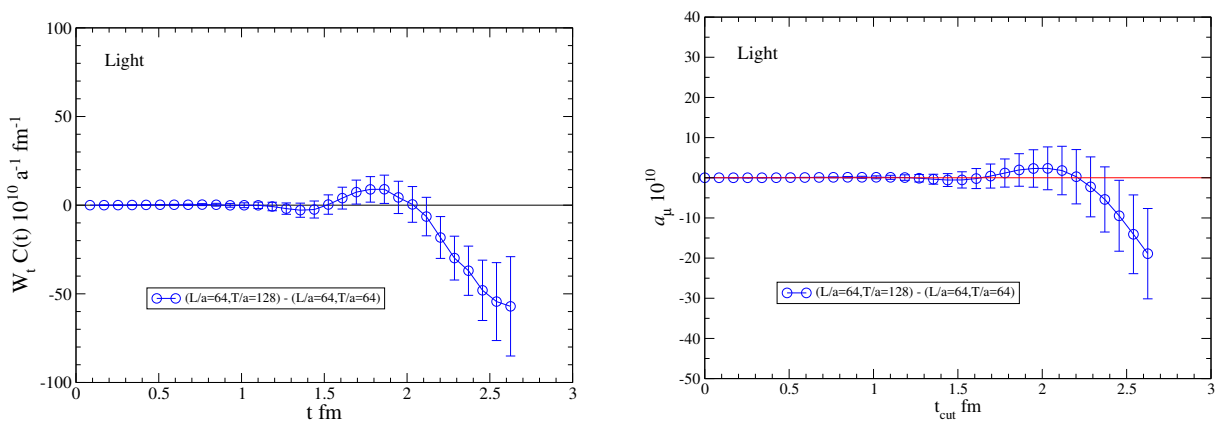

Figure 3. Difference of integrand divided by lattice spacing (left) and time-slice summation up to $t_{\text {cut }}$ from $L / a=64, T / a=128$ lattice to $L / a=64, T / a=64$ lattice at light quark flavor.

suffer the FV effect as positive contribution. This is due to extrapolation into $Q^{2}=0$ from large $Q^{2}$ point, for instance $Q^{2}=0.052 \mathrm{GeV}^{2}$ which is the minimum $Q^{2}$ on $64^{4}$ lattice. The peak position of the integrand (see Figure 4) has more than 10 times smaller than our minimum points, and it is thus a consequence of large uncertainty of $\mathrm{FV}$ effect missing in $Q^{2}$ integral between the minimum $Q^{2}$ point and peak position. Fortunately its effect is remedied on $96^{4}$ lattice, and in fact Figure 5 also indicated that two results in $Q^{2}$ integral and time-slice summation at light quark flavor becomes consistent within $1 \sigma$ error. Our study points out that in the physical pion, large lattice size as $L=8$ fm and more is needed to test consistency between both methods.

In our study, there are several systematics which has not been taken into account. First, since there is only one lattice cut-off scale on this ensemble, the lattice artifact effect involved into $a_{\mu}^{\text {hvp }}$ can not be measured directly. We however partially estimate it by comparison with the representation of $\left[\tilde{a}_{\mu}^{\text {hvp }}\right]_{\text {lat }}$ in Eq. (9). On such a way, lattice artifact correction appears in short time-slice, especially at $t / a=1$, and for the integral it thus affects constant shift. The magnitude of shift for $\left[a_{\mu}^{\text {hvp }}\right]_{\text {lat }}^{\text {light }}$ at $t_{\text {cut }}=3.02 \mathrm{fm}$ 

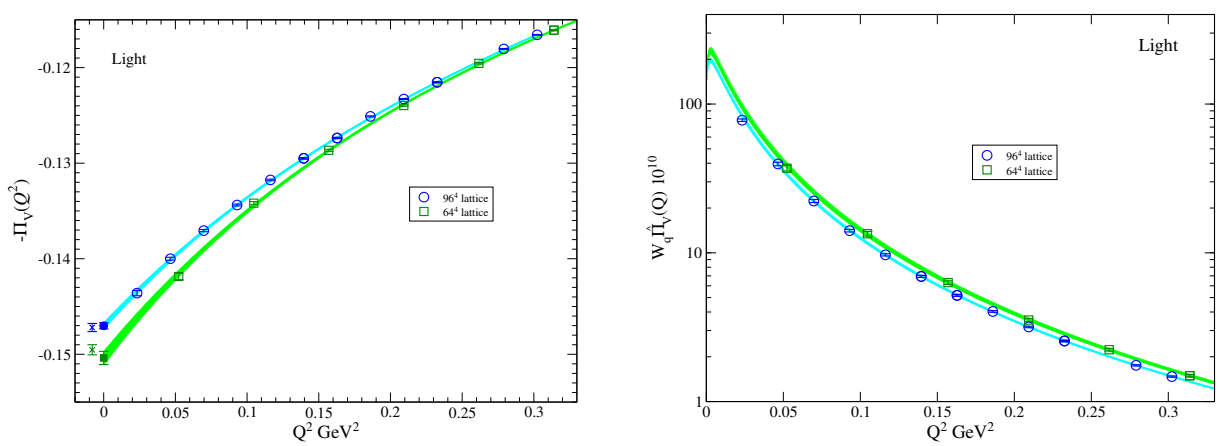

Figure 4. (Left) The $Q^{2}$ dependence of VPF extracted from Fourier transformed vector-vector current correlator at light quark flavor. Different symbols denote the data on different volumes. The curved band show the fitting function of Padé approximation with $[n, m]=[2,1]$ degree. The filled circle- and squared-symbol is an extrapolated result with Padé approximation. The cross-symbol is an extrapolated result with linear function using the lowest two $\mathrm{Q}^{2}$. (Right) This is a plot of integrand of $a_{\mu}^{\text {hvp }}$ in Eq.(1) as a function of $Q^{2}$. The data is obtained by subtraction of renormalization constant $\Pi(0)$ extrapolated by fitting function drawn in left figure. The curved band obtained from fitting function is a function for $Q^{2}$ integral. Symbols are same as left panel.

is,

$$
\left|\left[\tilde{a}_{\mu}^{\text {hvp }}\right]_{\text {lat }}^{\text {light }}-\left[a_{\mu}^{\text {hvp }}\right]_{\text {lat }}^{\text {light }}\right| \times 10^{10}=9.1(8.9),
$$

which is roughly $1.7 \%$ effect for total light flavor contribution. Note that this difference is only a consequence of discretized space-time on finite lattice spacing. The other lattice artifact caused by chiral symmetry breaking in Wilson-clover fermion should be estimated in the future using different cut-off scale. Second, as mentioned before, this is a calculation of only connected diagram, and the disconnected piece as SU(3) flavor symmetry breaking in electromagnetic current is other missing factor in our analysis. Although several papers [7, 22, 23] for computation of the disconnected piece in lattice QCD have reported a negative contribution to $a_{\mu}^{\text {hvp }}$ as $1.5 \%$, it will be tested on this ensemble in the next work.

\section{Acknowledgments}

Numerical calculations were performed on the $\mathrm{K}$ computer in RIKEN-AICS, Oakforest-PACS in JCAHPC, Hokusai at Advanced Center for Computing and Communication (ACCC) in RIKEN, XC40 at YITP in Kyoto University, and BlueGene/Q in High Energy Accelerator Research Organization (KEK). This computation is also supported by Interdisciplinary Computational Science Program No. xg17i019 in CCS, Large Scale Simulation Program No. 16/17-26 in KEK and General use No. G17029 at ACCC.

\section{References}

[1] G.W. Bennett et al. (Muon g-2), Phys. Rev. Lett. 92, 161802 (2004), hep-ex/0401008

[2] G.W. Bennett et al. (Muon g-2), Phys. Rev. D73, 072003 (2006), hep-ex/0602035 


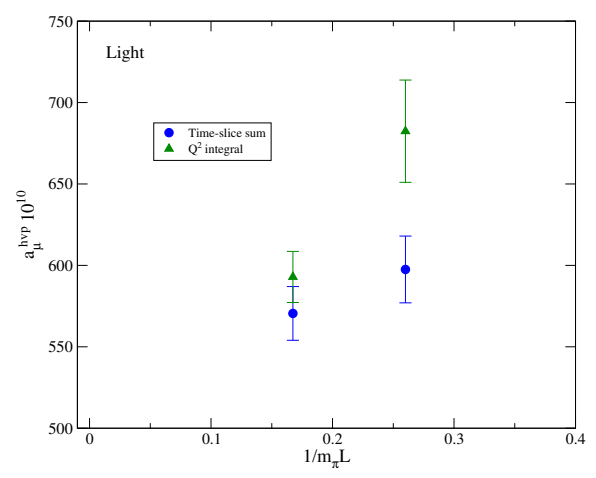

Figure 5. This is a plot of $m_{\pi} L$ dependence for $a_{\mu}^{\text {hvp }}$ obtained from two methods. "Time-slice sum" is a result of $\left[a_{\mu}^{\text {hvp }}\right]_{\text {lat }}$ " $Q^{2}$ integral" is a result of integral in momentum representation from zero to $Q^{2}=3.02 \mathrm{GeV}^{2}$ using $[2,1]$ Padé ansatz.

[3] T. Aoyama, M. Hayakawa, T. Kinoshita, M. Nio, Phys. Rev. Lett. 109, 111807 (2012), 1205.5368

[4] F. Jegerlehner, A. Nyffeler, Phys. Rept. 477, 1 (2009), 0902.3360

[5] D. Flay (Muon g-2), PoS ICHEP2016, 1075 (2017)

[6] K. Shimomura, Hyperfine Interact. 233, 89 (2015)

[7] M. Della Morte, A. Francis, V. Gülpers, G. Herdoíza, G. von Hippel, H. Horch, B. Jäger, H.B. Meyer, A. Nyffeler, H. Wittig (2017), 1705.01775

[8] G. von Hippel, T.D. Rae, E. Shintani, H. Wittig, Nucl. Phys. B914, 138 (2017), 1605.00564

[9] T. Blum, T. Izubuchi, E. Shintani, Phys.Rev. D88, 094503 (2013), 1208. 4349

[10] T. Blum, T. Izubuchi, E. Shintani, PoS LATTICE2012, 262 (2012), 1212 . 5542

[11] E. Shintani, R. Arthur, T. Blum, T. Izubuchi, C. Jung et al. (2014), 1402.0244

[12] B. Lautrup, A. Peterman, E. de Rafael, Physics Reports 3, 193 (1972)

[13] T. Blum, Phys. Rev. Lett. 91, 052001 (2003), hep-lat/0212018

[14] D. Bernecker, H.B. Meyer, Eur. Phys. J. A47, 148 (2011), 1107.4388

[15] E. Shintani, S. Aoki, H. Fukaya, S. Hashimoto, T. Kaneko et al., Phys.Rev. D82, 074505 (2010), 1002.0371

[16] P. Boyle, L. Del Debbio, E. Kerrane, J. Zanotti, Phys. Rev. D85, 074504 (2012), 1107 . 1497

[17] K.I. Ishikawa, N. Ishizuka, Y. Kuramashi, Y. Nakamura, Y. Namekawa, Y. Taniguchi, N. Ukita, T. Yamazaki, T. Yoshié (PACS), PoS LATTICE2015, 271 (2016), 1511.08549

[18] C. Aubin, T. Blum, M. Golterman, S. Peris, Phys. Rev. D86, 054509 (2012), 1205 . 3695

[19] K.I. Ishikawa, N. Ishizuka, Y. Kuramashi, Y. Nakamura, Y. Namekawa, Y. Taniguchi, N. Ukita, T. Yamazaki, T. Yoshie (PACS), PoS LATTICE2015, 075 (2016), 1511.09222

[20] M. Luscher, Comput.Phys.Commun. 156, 209 (2004), hep-lat/0310048

[21] M. Luscher, JHEP 0707, 081 (2007), 0706. 2298

[22] T. Blum, P.A. Boyle, T. Izubuchi, L. Jin, A. Jüttner, C. Lehner, K. Maltman, M. Marinkovic, A. Portelli, M. Spraggs, Phys. Rev. Lett. 116, 232002 (2016), 1512.09054

[23] S. Borsanyi, Z. Fodor, T. Kawanai, S. Krieg, L. Lellouch, R. Malak, K. Miura, K.K. Szabo, C. Torrero, B. Toth (2016), 1612 . 02364 